\title{
Giant Cell Granuloma due to Hyperparathyroidism: Case Report with Review of Litterature
}

\author{
Mohamed Yasser Ibrahim Daoud ${ }^{1 *}$, Ahmed Kheir ${ }^{3}$, Haytham M Alarfaj ${ }^{1}$, Aisha M Alzuhair ${ }^{1}$, Lena Kheir ${ }^{4}$, \\ Ahmed F Eid ${ }^{5}$, Hamed A AlWadaani ${ }^{1}$ and Ossama M Zakaria ${ }^{1,2}$
}

1 Department of Surgery, college of Medicine, King Faisal University, Al Ahsa, Kingdom of Saudi Arabia

${ }^{2}$ Department of Surgery, Faculty of Medicine, Suez Canal University, Egypt

${ }^{3}$ Sure Smile Dental, Scarsdale, New York, USA

${ }^{4}$ Rutgers Medical School, New Jersey, USA

${ }^{5}$ Medical Imaging Department, King Abdul-Aziz Hospital, Health Affairs of the Ministry of National Guard, Al-Ahsa, Kingdom of Saudi Arabia

*Corresponding author: Mohamed Yasser Ibrahim Daoud, Division of General Surgery, Department of Surgery, College of Medicine, King Faisal University, Kingdom of Saudi Arabia.
Received Date: June 23, 2020

Published Date: July 07, 2020

\begin{abstract}
Background: Giant cell granuloma associated with hyperparathyroidism is very limited and mostly has not been published in a wide scale before. Advances in laboratory techniques has resulted in the routine adoption of biomedical screening. This has led to the early diagnosis of hyperparathyroidism even with no symptoms. Moreover, advancement in radiological procedures has shown a great role in the diagnosis of many bony complications due to hyperparathyroidism.
\end{abstract}

Aim: The aim of this work is to present the development and presentations of giant cell granuloma in hyperparathyroidism.

Case presentation: The current study presented two cases of primary hyperparathyroidism that were discovered during a screening program out of 120 patients. These patients were clinically examined. There examination revealed a painless left mandibular swelling in one case and painful in the other which was diagnosed as periodontal abscess in one patient that was excluded from this study.

Conclusion: Hyperparathyroidism may be suspected when mandibular swelling is detected; the most effective therapy must be surgical excision with the control of the parathyroid level.

Keywords: Hyper-parathyroidism; Jaw lesions; Giant cell granuloma

\section{Introduction}

Regulation of the serum calcium level is governed by the Parathyroid hormone, yet calcium level itself may have an adverse effect in the control of the hormone secretion in a circular manner [1]. Hyper-parathyroidism may be clinically presented with the manifestations of an elevated parathormone level and hypercalceamia [2,3]. Hypercalcemia may also present with many clinical manifestations such as bony tumors of the maxillofacial area that are commonly presented in the mandible and rarely in the maxilla, among these tumors is the brown tumor that usually presents as well demarcated, circumscribed, osteolytic lesions within the jaws $[3,4]$. Their histopathological features are masses of soft tissue type compromising giant cells within the fibro vascular stroma, cyst like spaces line with connective tissue and foci of hemorrhage, micro fractures organized with the release of 
hemosiderin may also be noted, these findings appear as friable red brown masses, hence, the term brown tumors was used [3-7].

\section{Case Presentation}

A 52-year-old male who has not had a history of any chronic disease, presented with a large painless left mandibular swelling, fetid breath, intermittent intra-oral bleeding. He also suffered of difficulty in speaking, mastication and swallowing. His condition has started 8 weeks after extraction of the lower left wisdom tooth. The swelling showed a slowly continuous growth for a period of one year. His clinical examination revealed, a moderate left facial swelling, which was firm in consistency and slightly tender.

During the Intraoral examination, a sessile bluish mass that measured $7 \mathrm{~cm} \times 3 \mathrm{~cm} \times 2 \mathrm{~cm}$ was extending from the lower left first molar to the anterior border of the ramus obliterating the buccal sulcus and arising from the alveolar ridge, covered by normal pink mucosa (Figure 1). The mass was tender on palpation, firm in consistency, easily bleed on manipulation and has an indentation caused by the occlusion with the maxillary molar teeth. The overall dentition showed severe periodontal involvement manifested by gingival redness and bleeding gums with deep periodontal pockets.

The patient panoramic radiographs that showed a multilocular radiolucent lesion $6.5 \mathrm{~cm} \times 2 \mathrm{~cm}$ extending from the lower first molar area to involve the lower one third of left ramus, with resorption on distal surface of the first molar root. The lesion destructed buccal and lingual plates of mandibular alveolar bone. Other lesions were found involving the upper right first and second premolars (Figure 2). Follow up panoramic radiograph revealed more extension and involvement of the lower left part of body and ramus of the mandible with complete loss of roots of the lower left first molar and loss of the upper right second molar (Figure 3).

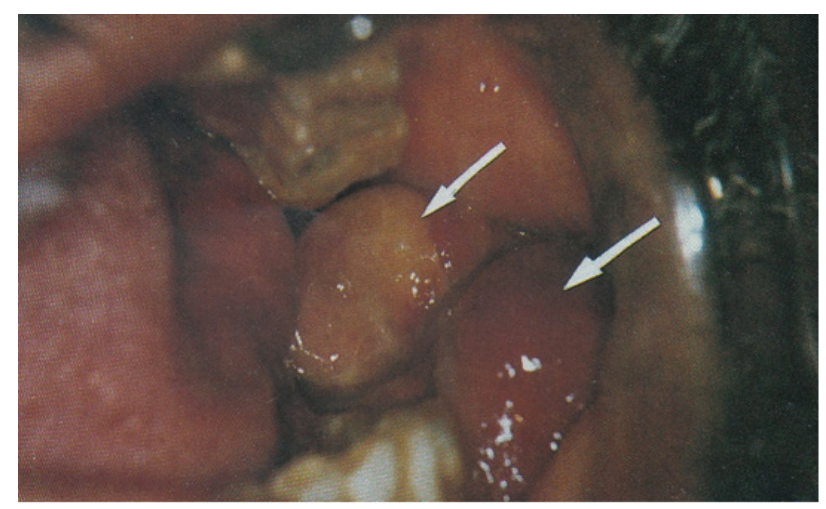

Figure 1: Intra-oral view of lower left alveolar ridge with two lobulated mass extending from the lower left first molar to anterior border of the ramus.

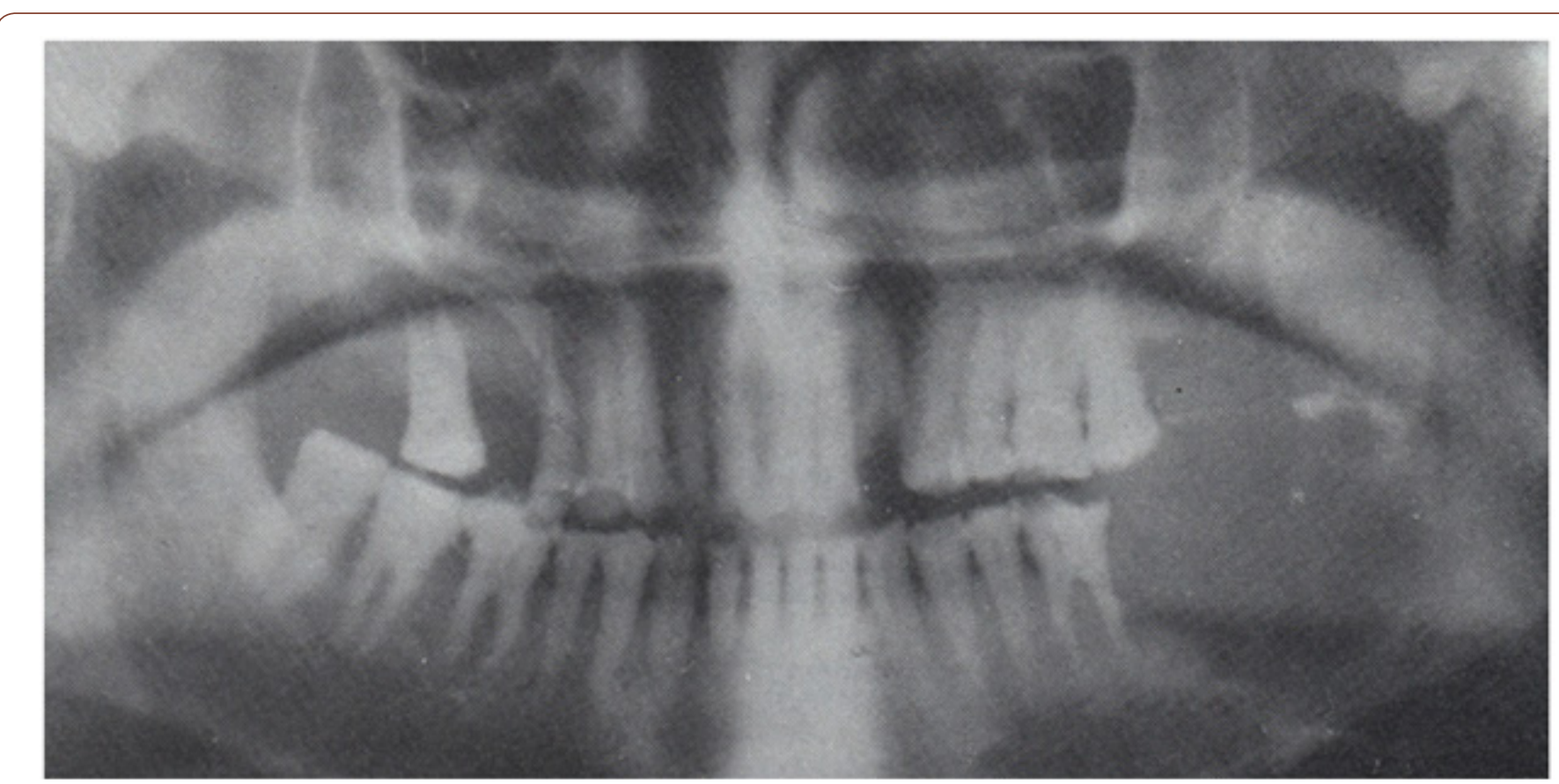

Figure 2: Panoramic radiograph of the case showing destruction of the buccal and lingual bone plates in the left mandibular region with resorption of the distal roots of the lower left first molar. 


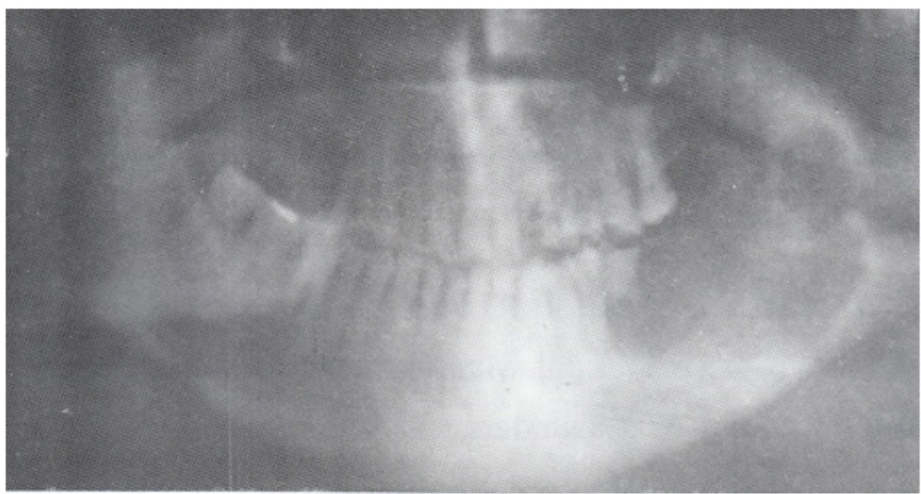

Figure 3: Updated panoramic radiograph showing more destruction of the left body and ramus and complete loss of the lower first molar roots

Following that CT scan was performed denoting the extension of the lesion to the submandibular region with destruction of buccal and lingual plates of bone. It also revealed affection of sub- mandibular region with the mass extending to the buccal soft tissues (Figure 4). Full body skeletal X-rays survey was unremarkable.

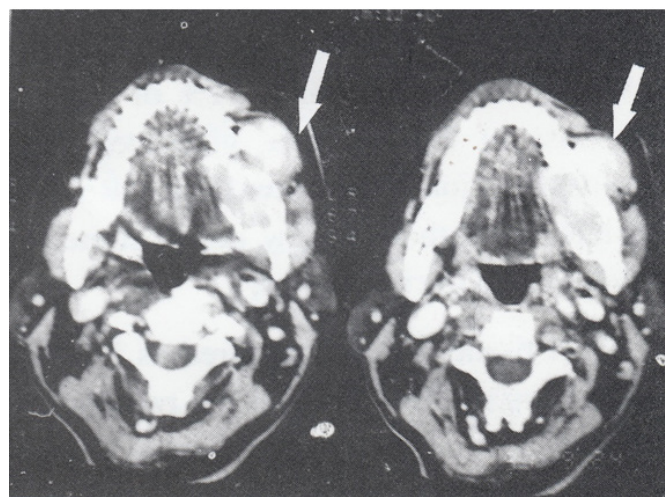

Figure 4: Axial CT scan of the head showing the extent of the lesion with involvement of the submandibular region and buccal soft tissue (arrows).

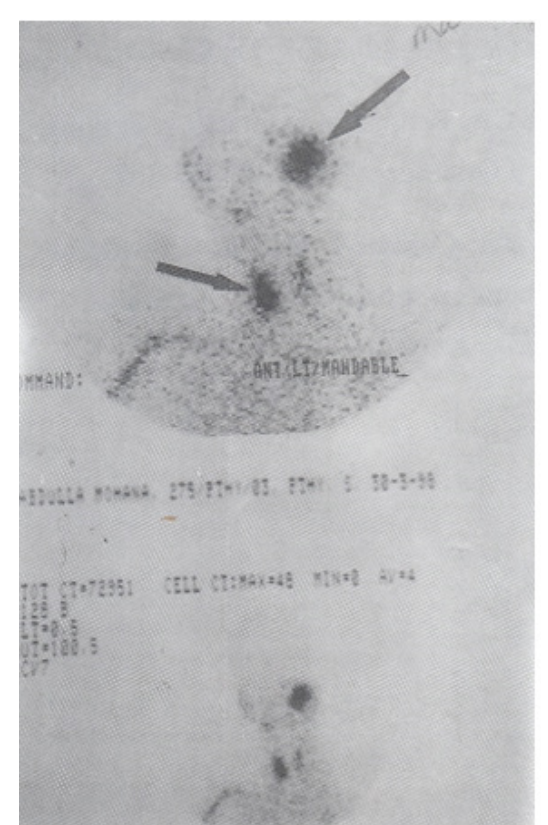

Figure 5: Isotope scan of the head and neck showing hot spot (arrow) or the left side of the face and right thyroid. 
The lesion incisional biopsy was histopathologicaly evaluated, revealing the presence of a stratified squamous epithelium alternating by focal areas of ulceration. However, the lesion underlying tissues had bundles of fibroblastic, spindle and polyhedral mesenchymal cells. It also revealed the presence of Variable number of multinucleated osteoclast-like giant cells that was having an intimate relationship to an ill-defined vascular space. Reported to be a peripheral giant cell granuloma (brown tumor).

The findings of the Initial routine laboratory tests were as follow:

\section{Serum calcium: $11.44 \mathrm{mg} / \mathrm{dl}$ (normal 8.1-10.4)}

Serum Phosphorus: $2.3 \mathrm{mg} / \mathrm{dl}$ (normal 2.7-4.5)

Alkaline Phosphatase: 285 IU/L (normal 50-279)

This was also associated with elevated level of serum PTH (it was $623 \mathrm{pg} / \mathrm{ml}$, normal $=9-55 \mathrm{pg} / \mathrm{ml}$ ).

Sonographic cervical assessment revealed a right thyroid lobe swelling of about $2 \mathrm{~cm}$ mass. Moreover, a hot spot was seen to be present within the left side of the mandible as well as the right thyroid lobe after radioactive isotope scans (Figure 5). Based on all the previously mentioned data the mass was preliminary diagnosed as primary hyper-parathyroidism that initially resembled intraoral peripheral giant cell granuloma.

Upon the Patient admission for surgery, his blood pressure was $240 / 110 \mathrm{~mm} / \mathrm{Hg}$, an ECG and echocardiograph reported cardiomyopathy; the patient underwent an excision of the right parathyroid mass with an intraoperative frozen section that revealed no malignancy. Yet his post-operative histopathology reported a parathyroid adenoma. Four days post-surgery, his serum calcium became normal and he was discharged after one week of uneventful post-operative course. He was appointed to be seen one month after discharge for further evaluation before excising the mandibular mass. Unfortunately, he was expired during this period with an obscure cause of death that was assumed to be of cardiac reasons.

Primary hyper-parathyroidism in many incidences may be asymptomatic, yet, the biomedical manifestations may be the only diagnostic tools [7].

Defects in calcium metabolism may lead to cystic bony changes [3]; in the current reported case, the bony lesions were restricted to the lower and upper jaws without involvement of any other bones. It has been concluded that minute percentage (7.1\%) of Primary hyper- parathyroidism-induced cystic bony lesions may exhibit giant loss of lamina Dura, giant cell lesions and demineralization of the jawbones. Others demonstrated 9.5\% involvement of the jaw bones $[8,9]$.
Soft tissue lesions appeared as lobulated mass causing intraoral swelling in the posterior region of the mandible, radiographic findings showed extensive resorption of the mandible, which suggests that the lesion started centrally and extended peripherally. Although it is tempting to speculate that they are only different anatomic manifestation of the same process, final proof is still lacking [7]. Our patient showed the peripheral lesion to be extensive, easily bled after the left lower wisdom tooth extraction, this coincides with the theory that trauma may be a crucial etiological factor in the appearance of such lesion.

\section{Conclusion}

This is a rare case of brown tumor induced by primary hyperparathyroidism. To our knowledge, this is the first case of a kind to be published. Although it is a single case, it may highlight the necessity of performing laboratory calcium and phosphorus profile before tooth extractions especially in the old people.

\section{Acknowledgement}

None.

\section{Conflict of Interest}

No conflict of interest.

\section{References}

1. Cebi Z, Keskin C, Guven Y (1989) Differences in the jaws and teeth of a patient with hypoparathyroidism. Dishekimliginde Klinik 2: 74-75.

2. Regezi J A and Sciubba J (1993) Oral Pathology Clinical Pathologic Correlations. WB Saunders pp: 461-464.

3. Killy HC, Kay LW (1965) Giant Cell Lesion of the jaws. J Int Coll Surg 44: 262 .

4. Auclair PL, Arendt DM, Hellstein JW (1997) Giant cell lesions of the jaws In: Gold Cleveland D (edt.), Oral and Maxillofacial Surgery Clinics of North America. Fibro-osseous diseases-considerations in diagnosis and management 9(7): 655-680.

5. Marx RE (2002) Fibro-osseous diseases and systemic diseases affecting bone. In: Marx RE, Stern D (edts.), Oral and maxillofacial pathology. A rationale for diagnosis and treatment. Chicago, IL: Quintessence Publishing Pp: 739-766

6. Parisien M, Silverberg SJ, Shane E, D W Dempster, J P Bilezikian, et al. (1990) Bone diseases in primary hyperparathyroidism. Endocrinol Metab Clin North Am 19: 18-34.

7. Rao P, Solomon M, Avramides A (1978) Brown tumors associated with secondary hyperparathyroidism of chronic renal failure. J Oral Surg 36: 154-159.

8. MacDonald DG, Lindsay R (1980) Skeletal disease, In JH Jones, DK Manson (edts.): Oral Manifestations of Systemic Diseases. WB Saunders pp: 381, London.

9. Rosenberg EH, Guralnick WC (1962) Hyperparathyroidism. A review of 220 proved cases with a special emphasis on findings in the jaws. Oral Surg (suppl) I5: 84-94. 